\title{
Effects of Roy Adaptation Model Education on Diabetes Care Profile of Patients with Type 2 Diabetes Mellitus
}

\author{
Brož Jan ${ }^{1, *}$, Mala Sarka ${ }^{1}$ and Rahelić Dario ${ }^{2}$ \\ ${ }^{1}$ Department of Internal Medicine, Second Faculty of Medicine, Charles University, Prague, Czech Republic \\ ${ }^{2}$ Department of Endocrinology, Diabetes and Clinical Pharmacology, Dubrava University Hospital, Zagreb, Croatia \\ "Corresponding author: Department of Internal Medicine, Second Faculty of Medicine, Charles University, Prague, Czech Republic, V Úvalu 84, 150 00, Prague, Czech Republic. \\ Tel: +420-22434001, Email: zorb@seznam.cz \\ Received 2018 July 30; Accepted 2018 September 07.
}

Keywords: Nursing, Roy Adaptation Model, Type 2 Diabetes

\section{Dear Editor,}

We have read with interest a paper by Kavuran and Yurttas entitled "Effects of Education Based on Roy Adaptation Model on Diabetes Care Profile of Patients with Type 2 Diabetes" (1) published recently in your journal. It is dealing with one of the currently used nursing models (the Roy adaptation model), which most importantly focuses on adaptation to a chronic disease (2). The model was also theoretically adapted for diabetes (3). The paper is presenting the outcomes of the study trying to evaluate the effects of educational intervention based on the Roy adaptation model on the psychosocial factors of diabetes care profile (factors that can aggravate or alleviate disease control). The results show improvements in the target group.

A comprehensive list of studies related to the topic and an interesting discussion have been provided in the paper. Nevertheless, we would like to ask the authors at least two questions.

1- Had the control group of patients ever experienced any type of education during the study period and why the study period differs between the experimental and control groups (three months vs. six months)?

2- Have you collected HbA1c values at the beginning and at the end of the study? Or would you just obtain them from the medical records? It would be interesting to see such data because they could show improvement also regarding metabolic control of disease and thus proof possible influence of your education to this very important area.

With great respect, we suggest taking into account these comments in the protocol of this interesting and important study, if its continuation is planned.

\section{Acknowledgments}

Special thanks to Rob Smith for language editing.

\section{Footnotes}

Conflict of Interests: The authors do not have any conflict of interests to declare.

Funding/Support: None declared.

\section{References}

1. Kavuran E, Yurttas A. Effects of education based on roy adaptation model on diabetes care profile of patients with type 2 diabetes. Iran Red Crescent Med J. 2018;20(4). e64635. doi: 10.5812/ircmj.64635.

2. Roy C. Extending the Roy adaptation model to meet changing global needs. Nurs Sci Q. 2011;24(4):345-51. doi: 10.1177/0894318411419210. [PubMed: 21975483].

3. Whittemore R, Sister Callista R. Adapting to diabetes mellitus: A theory synthesis. Nurs Sci Q. 2002;15(4):311-7. doi: 10.1177/089431802236796. [PubMed:12387229]. 